\title{
Plasma microRNAs are associated with acute exacerbation in idiopathic pulmonary fibrosis
}

Haiyan Min ${ }^{1,2}$, Shanshan Fan ${ }^{2}$, Shiyu Song ${ }^{2}$, Yi Zhuang ${ }^{1}$, Hui Li ${ }^{1}$, Yongzheng $\mathrm{Wu}^{2}$, Hourong Cai ${ }^{1}$, Long $\mathrm{Yi}^{2}$, Jinghong Dai ${ }^{1 *}$ and Qian Gao ${ }^{2^{*}}$

\begin{abstract}
Background: Acute exacerbation of idiopathic pulmonary fibrosis (AE-IPF) has high short-term mortality with unknown causes. To predict this malignant condition in clinics is challenging. In this study, we aim to demonstrate whether there are miRNAs that differ between AE-IPF and stable IPF, which may be served as reliable biomarker for AE-IPF prediction.

Methods: Human fibrotic-associated miRNAs arrays were designed to detect miRNAs expression in plasma of 3 AEIPF patients, 3 Stable-IPF (S-IPF) patients and 3 normal controls (NC). Differentially expressed miRNAs between AE-IPF and S-IPF patients were selected for further analyses. The validation studies were carried out in plasma of $12 \mathrm{AE}-\mathrm{IPF}$ patients, 45 S-IPF patients and 51 healthy control subjects. Signaling pathways and cellular processes interacted with validated miRNAs were predicted by DIANA-miRPath.

Results: According to the array analysis, 6 miRNAs showed differentiated expression between AE-IPF and S-IPF patients $(P<0.05)$. In the validation studies, let-7d-5p was decreased in S-IPF and further decreased in AE-IPF, when compared to NC $(0.0003 \pm 0.0002$ vs $0.003 \pm 0.002, P<0.01$ and $0.0007 \pm 0.0005$ vs $0.003 \pm 0.002, P<0.01)$. While miR- $25-3 p$ was obviously decreased in S-IPF $(0.0002 \pm 0.0001$ vs $0.0003 \pm 0.0003, P<0.01)$ but significantly increased in AE-IP $(0.0023 \pm$ 0.002 vs $0.0003 \pm 0.0003, P<0.01$ ). In receiver-operator characteristic $(\mathrm{ROC})$ curve analysis, the areas under the curve (AUCs) of miR-25-3p and let-7d-5p were 0.83 and 0.75 , respectively. The sensitivity at fixed specificity of $90 \%$ was improved from $50 \%$ to $66.7 \%$ when the two miRNAs were combined. The functional prediction of miRNAs suggested that the loss of anti-fibrotic capacity and the gain of uncontrolled cell growth may be required in AE-IPF pathogenesis. Conclusions: In conclusion, miR-25-3p and let-7d-5p in plasma were differentially expressed between AE-IPF and S-IPF. A combination of these two miRNAs may be a potential biomarker for AE-IPF from IPF.
\end{abstract}

Keywords: Idiopathic pulmonary fibrosis, Acute exacerbation, miR-25-3p, let-7d-5p

\section{Background}

Idiopathic pulmonary fibrosis (IPF) is a progressive disease with steady worsening of symptoms and gas exchange. Most patients die from respiratory failure within 3 years [1-3]. Although the clinical course of IPF is usually gradual, but persistent, decline in lung function, some patients may experience an acute worsening of dyspnea and

\footnotetext{
* Correspondence: daijinghongnew@sina.com; qian_gao@nju.edu.cn ${ }^{1}$ Department of Respiratory Medicine, Nanjing Drum Tower Hospital Affiliated to Medical School of Nanjing University, Nanjing, China ${ }^{2}$ Jiangsu Key Laboratory of Molecular Medicine, Medical School of Nanjing University, 22 Hankou Rd., Nanjing 210093, China
}

lung function with an unknown etiology [2], and is typically defined as acute exacerbations (AE) of IPF. AE-IPF may occur at any time during the disease course without an identifiable cause [1]. Approximately $50 \%$ of IPF patients died from $\mathrm{AE}[4]$ with a poor prognosis and a median survival of less than 3 months [5]. Current treatment strategies have consisted of high-dose corticosteroids, which has no any data from controlled trials to prove their efficacy for the patients of AE-IPF yet [5]. The pathological feature by chest high resolution computed tomography (HRCT) of AE-IPF is a diffuse alveolar damage superimposed on the usual interstitial pneumonia characteristics 
in IPF [6]. A better understanding of this disease is desirable.

Mature miRNAs are endogenous 18-24 nucleotide non-coding RNA molecules which act as posttranscriptional regulators of gene expression and are stable in circulation. The alterations of miRNAs in plasma or serum have been served as improved and noninvasive biomarkers for numerous pathological conditions, including cancers, autoimmune diseases and tissue injury [7-9]. Over the past years, molecular analysis of lung tissue resected for diagnostic purposes have provided more encouraging results that suggest that IPF lung biopsies have a unique mRNA transcriptome compared with non-fibrotic control biopsy samples $[10,11]$. More recently, much effort has been made to define biologically relevant transcriptional differences in IPF patients with distinguishable disease progressions [10,12]. Several studies have demonstrated that serum miRNAs were differentially expressed in IPF patients with rapidly or slowly progressive groups $[13,14]$. However, it is unknown whether differentially expressed miRNAs in the circulation is associated with AE-IPF.

In this retrospective study, we initially compared the human fibrosis-related miRNAs between S-IPF and AE-IPF. Moreover, through a validation study in an expanded cohort, we determined the predictive role of the miRNAs in distinguishing between S-IPF and AE-IPF.

\section{Methods}

\section{Study population}

The diagnosis of IPF was recommended by the American Thoracic Society and the European Respiratory Society (ATS $\backslash E R S)[15]$ and was previously described [16].

The diagnosis of AE-IPF was made according to the proposed diagnostic criteria that mainly focus on a previous or concurrent diagnosis of IPF with a worsening of its clinical manifestations over 30 days or less, and the absence of infection or another identifiable etiology such as left heart failure, or pulmonary embolism [17].

A total of 63 IPF patients were admitted to Drum Tower Hospital from January 1, 2013 to June 30, 2014. Among them, 15 patients were diagnosed as AE-IPF and died within 1 month after the diagnosis. And 48 IPF patients with the stable radiographic findings and without a worsening of their clinical manifestations over a period of 30 days were defined as S-IPF. These S-IPF patients had no acute exacerbation during the follow-up period of 12.5 years after enrollment. In addition, 54 unrelated agematched healthy individuals were enrolled without current or prior history of lung diseases in this study at the Center of Physical Examination of Drum Tower Hospital.

This study was approved by the Ethical Committee of the Affiliated Drum Tower Hospital of Nanjing University Medical School.

\section{Imaging studies}

Chest HRCT was performed using various CT scanners with the patients in suspended inspiration. The criteria for inclusion of IPF in the study described previously [16]. Chest HRCT for AE-IPF generally demonstrated bilateral ground-glass abnormality with or without areas of consolidation, superimposed on the UIP pattern described previously [2]. Two radiologists, without knowledge of any of the clinical, functional, and radiographic findings, independently reviewed the HRCT of all patients.

\section{Plasma miRNAs preparation}

Blood samples were obtained once the patients were diagnosed as S-IPF or AE-IPF. About $5 \mathrm{ml}$ venous blood samples were collected in EDTA-containing tubes. Blood samples were centrifuged within $1 \mathrm{~h}$ in two steps. Firstly, all samples were centrifuged at $800 \mathrm{~g}$ for $10 \mathrm{~min}$ at $4{ }^{\circ} \mathrm{C}$, and the supernatants were collected into new RNasefree tubes for a second centrifugation at 12,000 $\mathrm{g}$ for $10 \mathrm{~min}$ at $4{ }^{\circ} \mathrm{C}$. The supernatants were then stored for RNA extraction.

Plasma total RNA including miRNAs were extracted with miRNeasy Serum/Plasma Kit (Qiagen) from 400ul plasma samples according to the manufacturer's instructions. $100 \mathrm{pmol} / \mathrm{L}$ synthesized cel-miR-39 was added into plasma as an internal calibrator to monitor extraction efficiency. The concentration and purity of the total RNA were measured by Nanodrop 2000.

\section{miRNAs array procedure}

96-well miRNA expression profiles were used for $3 \mathrm{AE}$ IPF, 3 Stable-IPF(S-IPF) and 3 normal controls (NC). The array was designed according to miScript miRNA PCR Array (MIHS-117Z, Qiagen) including 84 human fibrosis-associated miRNAs, an external reference celmiR-39 and an internal reference U6. First, reverse transcription (RT) of the total RNA samples was performed with TaqMan miRNA Multiplex Reverse Transcription Kit (Applied Biosystems) according to its protocol. Next, quantitative real time PCR was performed according to the protocol of TaqMan Universal PCR master mix (Applied Biosystems) on Step One plus PCR system (Applied Biosystems).

\section{Quantitative RT-PCR (qRT-PCR) for plasma miRNAs validation}

Total miRNAs were extracted from $400 \mu \mathrm{l}$ plasma of additional 12 AE-IPF patients, 45 S-IPF patients, as well as 51 healthy controls with PARIS Kit (Ambion), mixed with $100 \mathrm{pmol} / \mathrm{L}$ synthesized cel-miR-39 as an internal calibrator to monitor extraction efficiency, and were dissolved in $50 \mu \mathrm{l}$ elution buffer. Total miRNAs were reverse transcribed into a final volume of $30 \mu \mathrm{L}$ cDNA with specific primers (Roche) using Prime Script RT- 
Table 1 Characteristics of study subjects

\begin{tabular}{|c|c|c|c|c|c|c|}
\hline & \multicolumn{3}{|l|}{ Array phase } & \multicolumn{3}{|c|}{ Validation phase } \\
\hline & $\begin{array}{l}\text { S-IPF } \\
(n=3)\end{array}$ & $\begin{array}{l}\text { AE-IPF } \\
(n=3)\end{array}$ & $\begin{array}{l}\text { Control } \\
(n=3)\end{array}$ & $\begin{array}{l}\text { S-IPF } \\
(n=45)\end{array}$ & $\begin{array}{l}\text { AE-IPF } \\
(n=12)\end{array}$ & $\begin{array}{l}\text { Control } \\
(n=51)\end{array}$ \\
\hline $\mathrm{Age}^{\mathrm{a}}$, mean $\pm \mathrm{SD}$ & $64.3 \pm 13.0$ & $63.7 \pm 9.7$ & $64.0 \pm 12.0$ & $63.9 \pm 11.7$ & $63.6 \pm 12.5$ & $65.9 \pm 9.7$ \\
\hline Female/Male & $1 / 2$ & $1 / 2$ & $1 / 2$ & $18 / 27$ & $5 / 7$ & $19 / 32$ \\
\hline $\mathrm{FVC}^{\mathrm{a}}(\%$ predicted), mean $\pm \mathrm{SD}$ & $63.7 \pm 17.2$ & $N A^{b}$ & NA & $65.7 \pm 10.7$ & $N A^{b}$ & NA \\
\hline $\mathrm{DL}_{\mathrm{CO}}^{\mathrm{a}}(\%$ predicted $)$, mean $\pm \mathrm{SD}$ & $56.3 \pm 19.1$ & $N A^{b}$ & NA & $51.2 \pm 17.7$ & $N A^{b}$ & NA \\
\hline
\end{tabular}

FVC forced vital capacity, DLCO diffusion capacity for carbon monoxide of lung, NA not available

${ }^{a}$ Values were collected at the diagnosis. ${ }^{b}$ patients were unable to be tested

PCR Kit (Takara) according to the instructions. Finally, quantitative PCR was performed with UPL probe kit (Roche) using Step One plus PCR system (Applied Biosystems). The reaction conditions were: $95{ }^{\circ} \mathrm{C}$ for $10 \mathrm{~min}$, and 40 cycles of $95^{\circ} \mathrm{C}$ for $15 \mathrm{~s}$ followed by $60^{\circ}$ $\mathrm{C}$ for $1 \mathrm{~min}$. Each sample was detected three times.

\section{MiRNAs target prediction and pathway analysis}

DIANA miRPath v.2.0 was used to predict target genes by one or more miRNAs into known KEGG pathways [18]. The graphical output of the program provides an overview of the parts of the pathway modulated by validated miRNAs. The statistical significance value associated with the identified signaling pathways and biological process was calculated by the program.

\section{Statistical analysis}

For the miRNA array and validation data analyses, the raw expression levels were determined by the cycle number at a predetermined threshold cycle $(\mathrm{CT})<35$. The foldchange for each miRNA from the control group to each patient group was calculated as $2^{-\Delta \Delta C T}$ with miR-16 as a reference miRNA. Relative Concentrations of miRNAs in AE-IPF, S-IPF and control plasma samples were calculated as $2^{-\triangle \mathrm{CT}}$ and compared by the T- test. Receiver-operator characteristic (ROC) curve was generated under the nonparametric distribution assumption for expression levels of plasma miRNAs of AE-IPF and S-IPF patients by plotting sensitivity\% vs (100\%-specificity \%). Areas under the curves (AUCs) were calculated. The biomarkers were considered to be useful when the value of AUCs were $>0.7$. Logistic regression was used in the combined markers analysis. All statistical analysis was performed with either SPSS17.0 or GraphPad Prism5.0 Software. 95\% confidence intervals were used to quantify uncertainty, and $P$ values $<0.05$ were considered to be statistically significant.

\section{Results}

\section{Patient characteristics}

Characteristics of patients and controls including age, gender, results of lung function tests were detailed in Table 1.
Identification of pulmonary fibrosis progress-related miRNAs in plasma

We initially profiled the expression of 84 fibrosis-related miRNAs in the plasma of 3 AE-IPF patients, 3 S-IPF patients and 3 healthy controls by the fibrosis-specific miRNAs arrays. With setting a threshold of CT $<35$, a total of 34 miRNAs were further analyzed. As shown in the heat map (Fig. 1), the levels of the majority of miRNAs detected in this study were decreased in both AEIPF and S-IPF patients, when compared to that of the controls. Among them, miR-132-3p and let-7d-5p were further decreased in AE-IPF patients when compared to that of S-IPF patients $(p<0.05)$. To identify the miRNAs that were deferentially expressed miRNAs between AEIPF and S-IPF patients, we further compared the expressions of the miRNAs in these two fibrotic groups to the

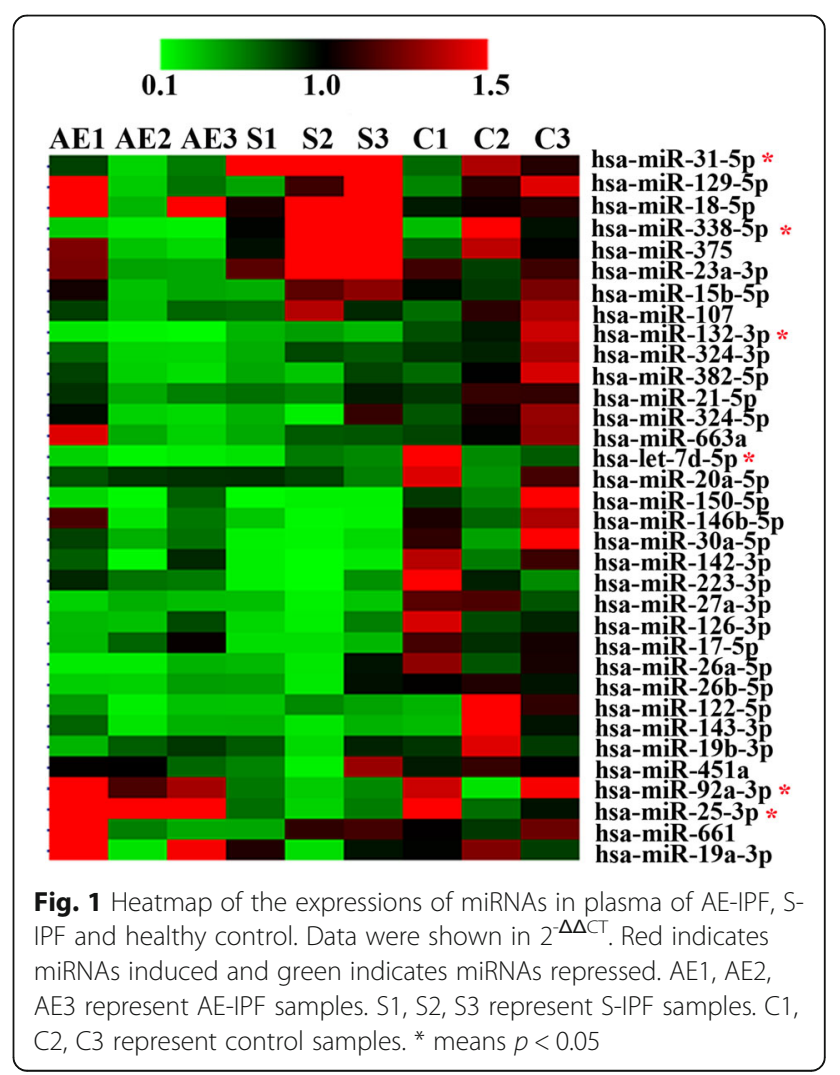



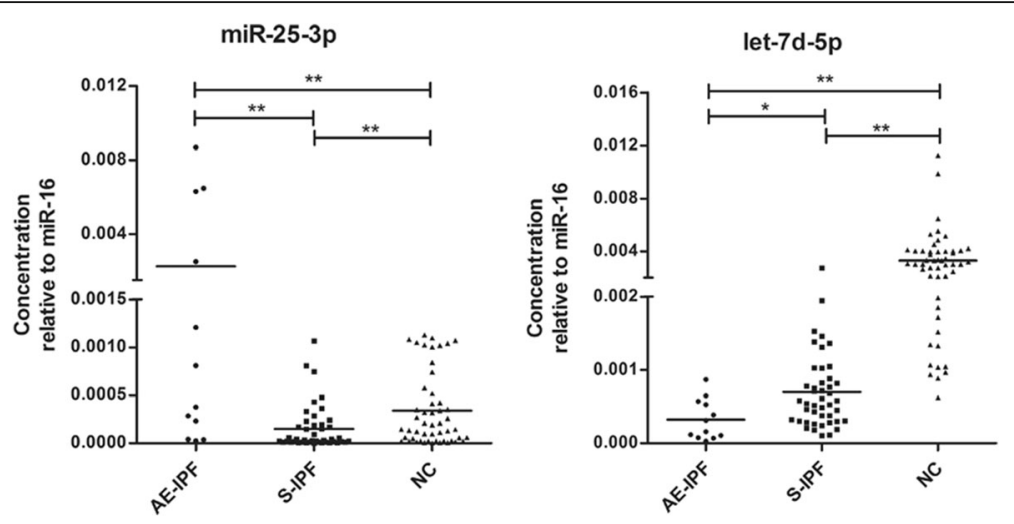

Fig. 2 Concentration of miR-25-3p and let-7d-5p in plasma of AE-IPF, S-IPF and healthy controls. The dash presents the mean value. NC, healthy controls. * means $p<0.05$. ** means $p<0.01$

control group. We found that miR-31-5p and miR-338$5 \mathrm{p}$ were up-regulated in S-IPF patients $(p<0.05)$, but down-regulated in AE-IPF patients $(p<0.05)$, while miR25-3p and miR-92-3p were up-regulated in AE-IPF group, but down-regulated in S-IPF group. All the above miRNAs were selected for further validation.

\section{Validation of differentially expressed miRNAs between AE-IPF and S-IPF}

Concentrations of the 6 miRNAs were detected in additional $12 \mathrm{AE}$-IPF and $45 \mathrm{~S}$-IPF patients, as well as 51 healthy controls by using RT-qPCR. We found that 4 of them, miR-132-3p, miR-338-5p, miR-92-3p and miR-31$5 p$, were not differentially expressed between AE-IPF and S-IPF groups (data not shown). As been shown in Fig. 2 and Table 2, the level of let-7d-5p was significantly decreased in AE-IPF patients, when compared to those of S-IPF patients $(p<0.05)$ and controls $(p<0.01)$. Interestingly, in the same comparison, miR-25-3p was found to be significantly decreased in S-IPF group $(p<0.01)$, but increased greatly in AE-IPF group $(p<0.01)$.

\section{ROC analysis}

To evaluate the effectiveness of the two miRNAs for predicting AE-IPF patients among IPF patients, ROC curves were constructed by comparing plasma measurements of let-7d-5p and miR-25-3p in the AE-IPF patients with that in the S-IPF patients. The diagnostic accuracy of the two miRNAs for AE-IPF prediction was calculated (Fig. 3 and Table 2). The AUCs of miR-25-3p and let-7d-5p were
0.831 and 0.752 , respectively. The two candidate markers were then subject to logistic regression analysis to optimize the diagnostic utility. The sensitivity at fixed specificity of $90 \%$ was improved from 50 to $66.7 \%$.

\section{Signaling pathways prediction}

DIANA-mirPath analysis was applied to predict the biologic targets and pathways as well as cellular processes that miR-25-3p and let-7d-5p affected. We found that the two miRNAs shared no common direct targets at transcriptional levels. We then investigated the cellular processes interacted with miR-25-3p and let-7d-5p. We found the intersections between the two miRNAs in cell cycle related processes in an opposite way, while let-7d$5 p$ showed the suppressive effects on these processes, miR-25-3p promoted (Table 3). Targets of miR-25-3p involved in TP53, BCL2L11 and CDKN1C, etc. The expressions of these genes were predicted to be downregulated by miR-25-3p in AE-IPF, which suggested a proliferative potential of cells. On the other hand, the targets of let-7d-5p included TGFBR1 and CDK6. The expressions of these genes were predicted to be upregulated in AE-IPF, which also suggested the probability of cells growth.

\section{Discussion}

During the last decade, several studies have explored biomarkers for the prediction of rapid progressive IPF [13, 14, 19-21]. Still, AE-IPF is a less characterized condition with unidentified causes and no tools for the

Table 2 Diagnostic sensitivities of measurements of single markers for predicting AE-IPF at predefined specificities

\begin{tabular}{|c|c|c|c|c|c|}
\hline & \multicolumn{3}{|c|}{ Relative concentration to miR-16 (mean \pm SD) } & \multirow[t]{2}{*}{$\mathrm{AUC}(95 \% \mathrm{Cl})$} & \multirow[t]{2}{*}{ Sensitivity at $90 \%$ Specificity } \\
\hline & $\mathrm{AE}-\mathrm{IPF}(n=12)$ & S-IPF $(n=45)$ & $\mathrm{NC}(n=51)$ & & \\
\hline miR-25-3p & $0.0023 \pm 0.0020$ & $0.0002 \pm 0.0001$ & $0.0003 \pm 0.0003$ & $0.83(0.70-0.96)$ & $50 \%$ \\
\hline let-7d-5p & $0.0003 \pm 0.0002$ & $0.0007 \pm 0.0005$ & $0.003 \pm 0.002$ & $0.75(0.59-0.91)$ & $50 \%$ \\
\hline miR-25-3p + let-7d-5p & - & - & - & $0.83(0.69-0.97)$ & $66.7 \%$ \\
\hline
\end{tabular}



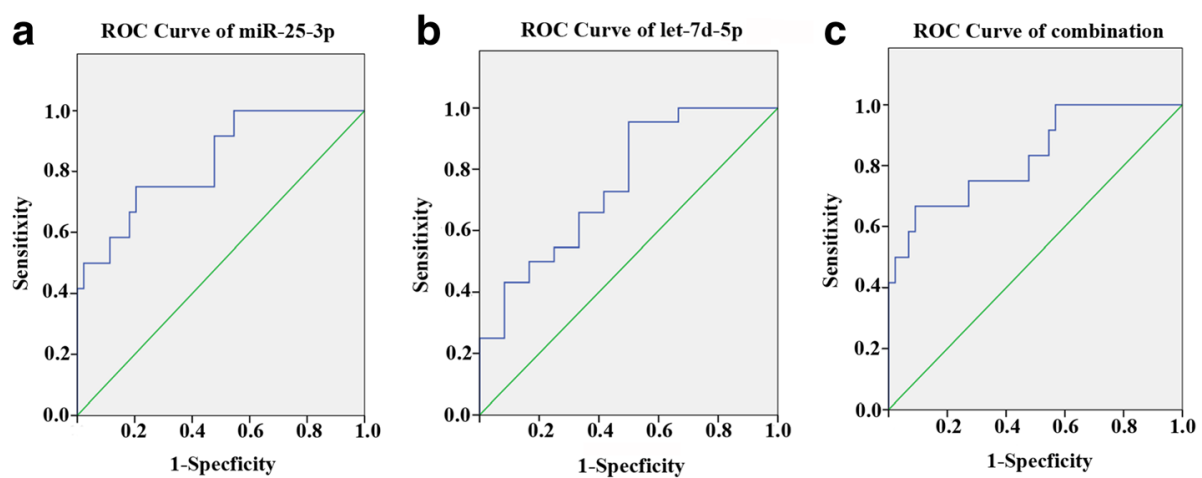

Fig. 3 ROC curve analyses of plasma miR-25-3p and let-7d-5p as single markers and combined markers for predicting AE-IPF from IPF. a ROC curve of miR-25-3p as a single biomarker for predicting AE-IPF from IPF. $\mathbf{b}$ ROC curve of let-7d-5p as a single biomarker for predicting AE-IPF from IPF. c ROC curve of combination of miR-25-3P and let-7d-5pas a biomarker for predicting AE-IPF from IPF

prediction. Current study offers an opportunity to appreciate circulating miRNAs detection as a newer standardized tool for prediction of AE-IPF.

In line with previous studies $[13,14]$, we found that let-7d-5p expression was decreased in the plasma of SIPF and further decreased in AE-IPF. We were also able to show that miR-25-3p was down-regulated in S-IPF, but significantly up-regulated in AE-IPF, a new observation that indicates that S-IPF and AE-IPF are indeed separable molecularly. As a single biomarker, the AUC of miR-25-3p or let-7d-5p was 0.831 or 0.752 respectively, and the sensitivity of the combination of the two at fixed specificity of $90 \%$ was significantly improved, arguing a beneficial potential with the combination of the two miRNAs in AE-IPF determination, although the AUC value of the current combination of the miRNAs isolated in our study is still relatively low. Further studies involve a larger AE-IPF cohort and a larger panel of testing miRNAs are desirable in order to obtain an improved AUC value. Since men and women are represented in the IPF population in different proportions, we compared the levels of miR-25-3p and let-7d-5p in the plasma of female and male patients in our study in both fibrotic groups, and observed no significant differences (data not shown).

The various functional studies on the two miRNAs, miR$25-3 p$ and let-7d-5p, should not be neglected. First, a reduction of let-7d in epithelial cells causes epithelialmesenchymal transition and over-expression of let-7d in fibroblasts can reduce their mesenchymal properties [22, 23], suggesting that the effect of let-7d is pro-fibrotic, a process occurring in both S-IPF and AE-IPF, but significantly enhanced in latter. On the other hand, miR-25 has been found to be increased in various cancers and may act as an oncomiRNA [24-28]. The specific large increase of miR-25-3p in AE-IPF, but reduced in S-IPF, suggested that the disease may have gained a pro-growth condition. Our findings have etiological implications that acute exacerbation in IPF may be due to the collapse of anti-fibrosis mechanisms and the gain of tumorigenic molecular tendency locally or systemically. Whether the two processes are parallel or mutually interacted is not known. More importantly, whether AE-IPF may obtain a neoplastic disease-like alteration is extremely provoking, but needs cellular and pathological evidence.

Table 3 The intersected biologic pathways of miR-25-3p and let-7d-5p predicted by KEGG pathways analysis

\begin{tabular}{llll}
\hline KEGG pathway & $p$-value & let-7-5p interacted genes & miR-25-3p interacted genes \\
\hline Glioma & $9.30 E-06$ & IGF1R,KRAS,CDK6,PDGFA & TP53 \\
Melanoma & $6.97 \mathrm{E}-05$ & IGF1R,KRAS,CDK6,PDGFA & TP53 \\
Chronic myeloid leukemia & 0.0002533 & IGF1R,KRAS,CDK6 & TP53 \\
Cell cycle & 0.0017125 & ESPL1,CDK6,BUB3,CDC25A & TP53,CDKN1C \\
Colorectal cancer & 0.0034595 & TGFBR1,BCL2,KRAS & TP53 \\
Pl3K-Akt signaling pathway & 0.0043839 & THBS1,BCL2,IGF1R,KRAS,CDK6,PDGFA & TP53,BCL2L11 \\
Hepatitis B & 0.0043839 & TGFBR1,BCL2,KRAS,CDK6 & TP53 \\
Pancreatic cancer & 0.0049791 & IGF1R,KRAS,CDK6 & TP53 \\
Bladder cancer & 0.0050961 & TGFBR1,KRAS & TP53 \\
Non-small cell lung cancer & 0.0060073 & KRAS,CDK6 & TP53 \\
HTLV-I infection & 0.0083159 & TGFBR1,KRAS,BUB3,ADCY9,PDGFA & TP53, AT2B \\
\hline
\end{tabular}


Interestingly, two genes, ATP11A and OBFC1 found to be associated with IPF susceptibility in a GWAS study [29] were predicted as targets of miR-25-3p. It will be highly interesting to study the association between the two genes and miR-25-3P in IPF pathogenesis. Moreover, it is known that the shortening of telomere length is a risk factor for the survival of IPF $[16,30]$. Whether one could make a link between the shortening of telomere length and the ill-expression of miRNAs in IPF progression remains to be determined.

In addition to the patients enrolled from a single medical center with a relatively small size in our study, one weakness of this investigation is that the current study was based on a scheme of one-time-point detection of the miRNAs without longitudinal measurements due to the clinical limitations, which may mask the relationship between the dynamic expressions of the miRNAs and the disease progression. Since the variability of the clinical courses of IPF are widely ranged and might be heterogeneous [31], the identification of the predictors that are capable of distinguishing the clinical courses of IPF would be a great challenge. Nevertheless, our study may have provided a clue to position miRNAs as the candidates of AE-IPF predicators.

\section{Conclusions}

In this study, we reported that the human fibrosisrelated miRNAs, miR-25-3p and let-7d-5p, were differentially expressed between S-IPF and AE-IPF. The combination of these two miRNAs was significant in predicting an acute exacerbation of IPF. The result indicates that both loss of tissue anti-fibrotic capacity and gaining of uncontrolled cell growth may be required in AE-IPF pathogenesis.

\section{Abbreviations}

AE-IPF: Acute exacerbation of idiopathic puhlmonary fibrosis; AUCs: Areas under the curves; IPF: Idiopathic pulmonary fibrosis; miRNAs: microRNAs; NC: Normal controls; ROC: Receiver-operator characteristic; S-IPF: Stable in idiopathic pulmonary fibrosis

\section{Acknowledgements}

We would like to thank all the participants in the studies.

\section{Funding}

The study was supported by the National Natural Science Foundation of China (81470253, 81570058, and 81570775), the Fundamental Research Funds for the Central Universities (021414340286), and the Medical Technology Project of Nanjing (YKK14063).

\section{Availability of data materials}

The datasets supporting the conclusions of this article are included within the article. Raw data files are available upon request.

\section{Authors' contributions}

HYM initially designed the study, carried out array analysis and RT-qPCR examinations, data analysis and drafted the manuscript. SSF performed samples extraction, the RT-qPCR examinations, and helped to data analysis. SYS contributed to array analysis and prepared the manuscript. YZ, HL and HRC helped to collect samples, HRCT examinations and lung function tests. YZW contributed to samples extraction and helped to draft the manuscript. LY helped to data analysis and functional prediction. JHD managed collection of samples and the clinical data, participated in study design. QG supervised, coordinated, and revised the manuscript. All authors read and approved the final manuscript.

\section{Competing interests}

The authors declare that they have no competing interests.

Consent for publication

Not applicable.

\section{Ethics approval and consent to participate}

This study was approved by the Ethical Committee of the Affiliated Drum Tower Hospital of Nanjing University Medical School (No. 2013081). All participants agreed to participate in this study at the time of blood samples collection.

Received: 22 July 2016 Accepted: 9 November 2016

Published online: 23 November 2016

\section{References}

1. Kim DS, Collard HR, King TJ. Classification and natural history of the idiopathic interstitial pneumonias. Proc Am Thorac Soc. 2006;3:285-92.

2. Collard HR, Moore BB, Flaherty KR, Brown KK, Kaner RJ, King TJ, Lasky JA, Loyd JE, Noth I, Olman MA, et al. Acute exacerbations of idiopathic pulmonary fibrosis. Am J Respir Crit Care Med. 2007;176:636-43.

3. Hyzy R, Huang S, Myers J, Flaherty K, Martinez F. Acute exacerbation of idiopathic pulmonary fibrosis. Chest. 2007;132:1652-8.

4. Natsuizaka M, Chiba H, Kuronuma K, Otsuka M, Kudo K, Mori M, Bando M, Sugiyama Y, Takahashi $H$. Epidemiologic survey of Japanese patients with idiopathic pulmonary fibrosis and investigation of ethnic differences. Am J Respir Crit Care Med. 2014;190:773-9.

5. Song JW, Hong SB, Lim CM, Koh Y, Kim DS. Acute exacerbation of idiopathic pulmonary fibrosis: incidence, risk factors and outcome. Eur Respir J. 2011;37:356-63.

6. Parambil JG, Myers JL, Ryu JH. Histopathologic features and outcome of patients with acute exacerbation of idiopathic pulmonary fibrosis undergoing surgical lung biopsy. Chest. 2005;128:3310-5.

7. Mitchell PS, Parkin RK, Kroh EM, Fritz BR, Wyman SK, Pogosova-Agadjanyan EL, Peterson A, Noteboom J, O'Briant KC, Allen A, et al. Circulating microRNAs as stable blood-based markers for cancer detection. Proc Natl Acad Sci U S A. 2008;105:10513-8.

8. Tijsen AJ, Pinto YM, Creemers EE. Circulating microRNAs as diagnostic biomarkers for cardiovascular diseases. Am J Physiol Heart Circ Physiol. 2012; 303:H1085-95.

9. Laterza OF, Lim L, Garrett-Engele PW, Vlasakova K, Muniappa N, Tanaka WK, Johnson JM, Sina JF, Fare TL, Sistare FD, et al. Plasma MicroRNAs as sensitive and specific biomarkers of tissue injury. Clin Chem. 2009;55:1977-83.

10. Selman M, Pardo A, Barrera L, Estrada A, Watson SR, Wilson K, Aziz N, Kaminski N, Zlotnik A. Gene expression profiles distinguish idiopathic pulmonary fibrosis from hypersensitivity pneumonitis. Am J Respir Crit Care Med. 2006:173:188-98.

11. Yang IV, Burch LH, Steele MP, Savov JD, Hollingsworth JW, McElvaniaTekippe E, Berman KG, Speer MC, Sporn TA, Brown KK, et al. Gene expression profiling of familial and sporadic interstitial pneumonia. Am J Respir Crit Care Med. 2007;175:45-54

12. Boon K, Bailey NW, Yang J, Steel MP, Groshong S, Kervitsky D, Brown KK, Schwarz MI, Schwartz DA. Molecular phenotypes distinguish patients with relatively stable from progressive idiopathic pulmonary fibrosis (IPF). PLoS One. 2009;4:e5134

13. Oak SR, Murray L, Herath A, Sleeman M, Anderson I, Joshi AD, Coelho AL, Flaherty KR, Toews GB, Knight D, et al. A micro RNA processing defect in rapidly progressing idiopathic pulmonary fibrosis. PLoS One. 2011;6:e21253.

14. Yang G, Yang L, Wang W, Wang J, Wang J, Xu Z. Discovery and validation of extracellular/circulating microRNAs during idiopathic pulmonary fibrosis disease progression. Gene. 2015;562:138-44.

15. American Thoracic Society. Idiopathic pulmonary fibrosis: diagnosis and treatment. International consensus statement. American Thoracic Society (ATS), and the European Respiratory Society (ERS). Am J Respir Crit Care Med. 2000;161:646-64 
16. Dai J, Cai H, Zhuang Y, Wu Y, Min H, Li J, Shi Y, Gao Q, Yi L. Telomerase gene mutations and telomere length shortening in patients with idiopathic pulmonary fibrosis in a Chinese population. Respirology. 2015;20:122-8.

17. Raghu G, Collard HR, Egan JJ, Martinez FJ, Behr J, Brown KK, Colby TV, Cordier JF, Flaherty KR, Lasky JA, et al. An official ATS/ERS/JRS/ALAT statement: idiopathic pulmonary fibrosis: evidence-based guidelines for diagnosis and management. Am J Respir Crit Care Med. 2011;183:788-824.

18. I. S. Vlachos NKTV. DIANA miRPath v.2.0: investigating the combinatorial effect of microRNAs in pathways. Nucleic Acids Res. 2012;40:W498-504.

19. Matsuzawa Y, Kawashima T, Kuwabara R, Hayakawa S, Irie T, Yoshida T, Rikitake H, Wakabayashi T, Okada N, Kawashima K, et al. Change in serum marker of oxidative stress in the progression of idiopathic pulmonary fibrosis. Pulm Pharmacol Ther. 2015;32:1-6.

20. Kakugawa T, Yokota S, Ishimatsu Y, Hayashi T, Nakashima S, Hara S, Sakamoto N, Kubota H, Mine M, Matsuoka Y, et al. Serum heat shock protein 47 levels are elevated in acute exacerbation of idiopathic pulmonary fibrosis. Cell Stress Chaperones. 2013;18:581-90.

21. Asano S, Takemura T, Katoh K, Taneda M, Kitagawa M. Epithelial regeneration after diffuse alveolar damage in relation to underlying disease and DAD stage: an autopsy study. J Med Dent Sci. 2011;58:113-21.

22. Pandit KV, Corcoran D, Yousef H, Yarlagadda M, Tzouvelekis A, Gibson KF, Konishi K, Yousem SA, Singh M, Handley D, et al. Inhibition and role of let$7 \mathrm{~d}$ in idiopathic pulmonary fibrosis. Am J Respir Crit Care Med. 2010;182:220-9.

23. Huleihel L, Ben-Yehudah A, Milosevic J, Yu G, Pandit K, Sakamoto K, Yousef $\mathrm{H}$, LeJeune $\mathrm{M}$, Coon TA, Redinger $\mathrm{CJ}$, et al. Let-7d microRNA affects mesenchymal phenotypic properties of lung fibroblasts. Am J Physiol Lung Cell Mol Physiol. 2014;306:L534-42.

24. Li LM, Hu ZB, Zhou ZX, Chen X, Liu FY, Zhang JF, Shen HB, Zhang CY, Zen K. Serum microRNA profiles serve as novel biomarkers for HBV infection and diagnosis of HBV-positive hepatocarcinoma. Cancer Res. 2010;70:9798-807.

25. Zhao H, Wang Y, Yang L, Jiang R, Li W. MiR-25 promotes gastric cancer cells growth and motility by targeting RECK. Mol Cell Biochem. 2014;385:207-13.

26. Wang X, Meng X, Li H, Liu W, Shen S, Gao Z. MicroRNA-25 expression level is an independent prognostic factor in epithelial ovarian cancer. Clin Trans Oncol. 2014;16:954-8.

27. Razumilava N, Bronk SF, Smoot RL, Fingas CD, Werneburg NW, Roberts LR, Mott JL. miR-25 targets TNF-related apoptosis inducing ligand (TRAIL) death receptor-4 and promotes apoptosis resistance in cholangiocarcinoma. Hepatology. 2012;55:465-75.

28. Li BS, Zuo QF, Zhao YL, Xiao B, Zhuang Y, Mao XH, Wu C, Yang SM, Zeng $H$ Zou QM, et al. MicroRNA-25 promotes gastric cancer migration, invasion and proliferation by directly targeting transducer of ERBB2, 1 and correlates with poor survival. Oncogene. 2015:34:2556-65.

29. Fingerlin TE, Murphy E, Zhang W, Peljto AL, Brown KK, Steele MP, Loyd JE, Cosgrove GP, Lynch D, Groshong S, et al. Genome-wide association study identifies multiple susceptibility loci for pulmonary fibrosis. Nat Genet. 2013; 45:613-20.

30. Alder JK, Chen JJ, Lancaster L, Danoff S, Su SC, Cogan JD, Vulto I, Xie M, Qi $\mathrm{X}$, Tuder RM, et al. Short telomeres are a risk factor for idiopathic pulmonary fibrosis. Proc Natl Acad Sci U S A. 2008;105:13051-6.

31. Moore BB, Fry C, Zhou Y, Murray S, Han MK, Martinez FJ, Flaherty KR. Inflammatory leukocyte phenotypes correlate with disease progression in idiopathic pulmonary fibrosis. Front Med. 2014;1:1-12.

\section{Submit your next manuscript to BioMed Central and we will help you at every step:}

- We accept pre-submission inquiries

- Our selector tool helps you to find the most relevant journal

- We provide round the clock customer support

- Convenient online submission

- Thorough peer review

- Inclusion in PubMed and all major indexing services

- Maximum visibility for your research

Submit your manuscript at www.biomedcentral.com/submit

) Biomed Central 